\title{
ROS1 in Squamous Non-Small Cell Lung Cancer-Combined Immunotherapy (PD1/CTLA4) or Targeted Therapy?
}

\author{
Alexander Yakobson ${ }^{*}$, Tal Mor ${ }^{2 *}$, Levitas Dina1, Laila C. Roisman1, Daniel Levin ${ }^{3}$, \\ Wafeek Alguayn' ${ }^{1}$, Sara Morgenstern ${ }^{4}$, Keren Rouvinov ${ }^{1}$, Nir Peled ${ }^{1 \#, ~ W a l e e d ~ K i a n}{ }^{1}$ \\ ${ }^{1}$ The Legacy Heritage Oncology Center \& Dr. Larry Norton Institute, Soroka Medical Center \& Ben-Gurion University, Beer-Sheva, Israel \\ ${ }^{2}$ Sackler Faculty of Medicine, Tel Aviv University, Tel Aviv, Israel \\ ${ }^{3}$ Faculty of Health Sciences, Ben-Gurion University of the Negev, Beer-Sheva, Israel \\ ${ }^{4}$ Rabin Medical Center, Beilinson Campus, Petah Tikva, Israel \\ Email: "peled.nir@gmail.com, YamitBY@clalit.org.il
}

How to cite this paper: Yakobson, A., Mor, T., Dina, L., Roisman, L.C., Levin, D., Alguayn, W., Morgenstern, S., Rouvinov, K., Peled, N. and Kian, W. (2020) ROS1 in Squamous Non-Small Cell Lung CancerCombined Immunotherapy (PD1/CTLA4) or Targeted Therapy?. Journal of Cancer Therapy, 11, 365-370.

https://doi.org/10.4236/jct.2020.116031

Received: April 17, 2020

Accepted: June 8, 2020

Published: June 11, 2020

Copyright $\odot 2020$ by author(s) and Scientific Research Publishing Inc. This work is licensed under the Creative Commons Attribution International License (CC BY 4.0).

http://creativecommons.org/licenses/by/4.0/ (c) (i) Open Access

\begin{abstract}
ROS1 oncogenic fusion is reported to be $1 \%-2 \%$ of non-small cell lung cancers (NSCLCs) of the adenocarcinoma subgroup. Meanwhile, there are no records of squamous cell cancer patients with tumors harboring ROS1 fusions. The Foundation Medicine database indicates a frequency of ROS1 rearrangements is $0.2 \%$ among squamous NSCLC. Crizotinib is known to be very effective in these patients. Here we present a non-smoker patient who had pure squamous NSCLC that was treated by combinational immunotherapy under a clinical trial and progressed after 2 cycles. Surprisingly, comprehensive genomic profiling detected a rare oncogenic EZR-ROS1 fusion, and the patient was treated by crizotinib with a significant response within 6 weeks. To date, the patient has been on therapy for 42 months and has achieved a complete metabolic response.
\end{abstract}

\section{Keywords}

ROS1, Crizotinib, Squamous Cell Lung Cancer, Immunotherapy; Non-Small Cell Lung Cancer

\section{Introduction}

ROS1 oncogenic fusion is reported to be $1 \%-2 \%$ of non-small cell lung cancers (NSCLCs) of the adenocarcinoma subgroup [1]. Concurrently, the COSMIC database indicates that only $5.33 \%$ of squamous cell cancer (SCC) patients have

*These two authors contributed equally to this work. 
single point mutations in the ROS1 gene (53/995) and the vast majority of which are of unknown functional significance. Meanwhile, there are no records of SCC patients with tumors harboring ROS1 fusions [2]. The Foundation Medicine database indicates a frequency of ROS1 rearrangements in $1.1 \%(181 / 16,878)$ and $0.2 \%(5 / 2,386)$, respectively, among non-squamous NSCLC and squamous NSCLC. Crizotinib is known to be very effective in ROS1-positive patients and is Food and Drug Administration (FDA) approved for the treatment of patients with advanced ROS1-positive NSCLC [3]. Several other tyrosine kinase inhibitors (TKI) have been approved later on, for example Ceritinib and Lorlatinib [4]. Pembrolizumab alone or in combination with chemotherapy is considered first line therapy for non-small-cell lung cancer (NSCLC) [5] [6] [7]. Immuno-oncology (IO) combination therapy, for example Nivolumab-Ipilimumab, has also been tested as 1st line in NSCLC, in which therapy targets both PD-1 and CTLA4 [8]. This combination IO showed better progression-free survival (PFS) compared to anti-PD1 single agent (CheckMate 227) and particularly in patients with low PD-L1 expressions and a high tumor mutation burden. In patients presenting with a driver mutation and specifically Epidermal Growth Factor Receptor and Anaplastic Lymphoma Kinase (EGFR/ALK) alterations, the effectiveness of IO monotherapy is low [9], and the role of IO-IO combination is yet unknown.

Here we present a non-smoker patient who had pure squamous NSCLC that was treated by CTLA4 \& PD-L1 combinational immunotherapy under a clinical trial and progressed after 2 cycles. Surprisingly, comprehensive genomic profiling detected a rare oncogenic EZR-ROS1 fusion, and the patient was treated by crizotinib with a significant response within 6 weeks. To date, the patient has been on therapy for 42 months and has achieved a complete metabolic response.

\section{Case Presentation}

In September 2016, a non-smoking 63-year-old female presented to the emergency room due to shortness of breath. A chest $\mathrm{x}$-ray demonstrated right pleural effusion (Figure 1). Diagnostic pleural drainage was performed, cell block from the pleural fluid showed small groups of malignant epithelial cells with squamoid appearance (Figure 2). A positron emission tomography-computed tomography (PET-CT) demonstrated a high uptake of FDG in the right upper lobe (RUL), liver and bone (Figure 3(a)). A palliative radiotherapy to the RUL consisted of $30 \mathrm{~Gy}$ in 10 fractions was initiated. Subsequently, a systemic first line combination therapy with anti PD-L1 and CTLA4 inhibitors were initiated under a clinical trial (Durvalumab + tremelimumab) for 2 cycles with significant clinical disease progression, which was confirmed by computed tomography (CT) scan (Figure 3(b)). Genomic profiling conducted by (FoundationOne, Foundation Medicine, Cambridge, MA) showed an oncogenic EZR (exons 1 9)-ROS1 (exons 34 - 43) fusion with a low TMB (4.79 mutations/Megabase). Crizotinib was started immediately with a significant clinical improvement within 6 weeks and subsequently achieved a complete metabolic response lasting, 
to date, for 42 months (Figure 3(c)).

\section{Discussion}

Here we describe a pure squamous NSCLC in a non-smoker patient that was treated by combination immunotherapy with rapid disease progression after 2 cycles. A genomic profiling detected a rare oncogenic EZR-ROS1 fusion. Subsequently,

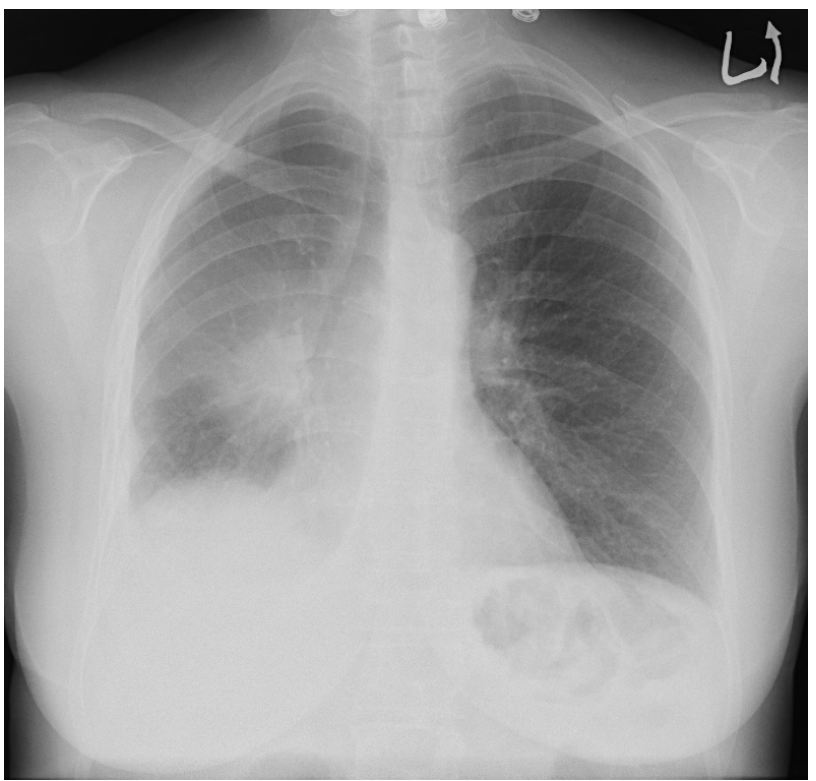

Figure 1. Chest X-ray demonstrated right pleural effusion and solid lesion.
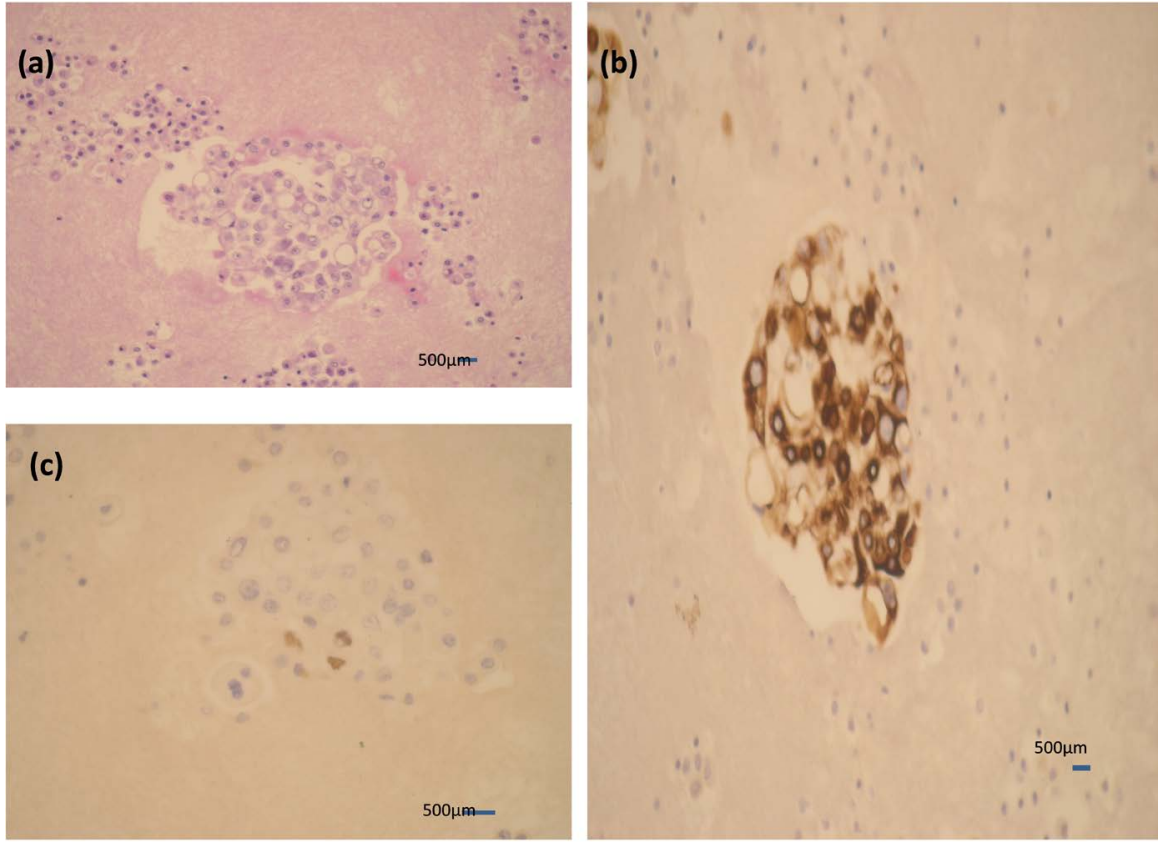

Figure 2. H \& E staining cell block performed from the pleural fluid shows small groups of malignant epithelial cells with squamoid appearance (a). On the background, mesothelial cells and lymphocytes. The immunohistochemical stain for keratin 5/6 shows diffuse positivity (b). The P63 stains a few cells only (c). 
(a)

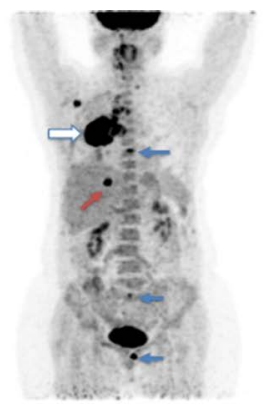

(b)

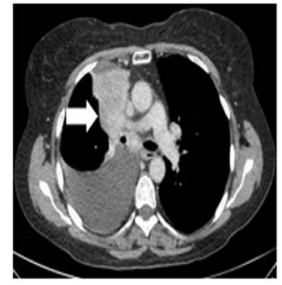

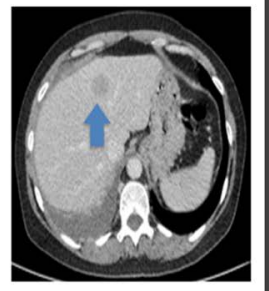

(c)

Figure 3. Whole body imaging $F^{8}$-fluorodeoxyglucose PET-CT showing (a) large lung mass (white arrow) with multiple mediastinal lymph nodes, liver lesion (red arrow) and multiple bone lesions (blue arrow). (b) Restaging computed tomography demonstrating stable Lung mass (white arrow) and increase in size of liver lesion (blue arrow). (c) Follow up PET-CT demonstrating no substantial pathological uptake in previous known site of disease.

the patient was treated by crizotinib with a significant response within 6 weeks. Currently, 42 months since starting the treatment with achieved complete metabolic response.

Oncogenic drivers in NSCLC adenocarcinoma are particularly common amongst the non-smoking population which broadly concords with this rare non-smoking squamous cell cancer patient with a ROS1 fusion. To our knowledge, this is the first published case of a ROS1 fusion identified in a pure squamous cell lung carcinoma. In general, the presence of gene rearrangement of RET, ALK and ROS in SCC is considered very rare.

This SCC patient rapidly progressed on immune-checkpoint inhibitors without having a known genomic profile, whilst harboring a ROS1 fusion mutation. Consequently, it is plausible to think that treatment better suited for this patient was unfortunately delayed, which is demonstrated by the impressive response to crizotinib. This case reconfirms the need for upfront genomic profiling in all NSCLC including never-smokers SCC for potentially harboring a possibly treatable driver mutation as demonstrated. This case may also indicate that patients with tumors harboring ROS1 fusions are less likely to benefit from immunotherapy, even in a combinational regimen.

\section{Author Contribution}

Conception and design: NP, WK, TM. Manuscript writing: All authors. Final 
approval of manuscript: All authors.

\section{Acknowledgments}

We would like to thank the patient and the patient's family for their cooperation during the whole treatment. Patient's informed consent has been obtained for the publication of the finding and figures presented in this report.

\section{Ethical Statement}

The authors are accountable for all aspects of the work in ensuring that questions related to the accuracy or integrity of any part of the work are appropriately investigated and resolved.

\section{Conflicts of Interest}

The authors declare no conflicts of interest regarding the publication of this paper.

\section{References}

[1] Lin, J.J., Ritterhouse, L.L., Ali, S.M., et al. (2017) ROS1 Fusions Rarely Overlap with Other Oncogenic Drivers in Non-Small Cell Lung Cancer. Journal of Thoracic Oncology, 12, 872-877. https://doi.org/10.1016/j.jtho.2017.01.004

[2] Zhao, W., Choi, Y.L., Song, J.Y., et al. (2016) ALK, ROS1 and RET Rearrangements in Lung Squamous Cell Carcinoma Are Very Rare. Lung Cancer, 94, 22-27. https://doi.org/10.1016/j.lungcan.2016.01.011

[3] Shaw, A.T., Ou, S.H.I., Bang, Y.J., et al. (2014) Crizotinib in ROS1-Rearranged Non-Small-Cell Lung Cancer [Internet]. New England Journal of Medicine, 371, 1963-1971. http://www.nejm.org/doi/abs/10.1056/NEJMoa1406766

[4] Facchinetti, F. and Friboulet, L. (2018) Lorlatinib in ALK- and ROS1-Positive NSCLC: The Future Has a Start. Translational Lung Cancer Research, 7, S103-S106. https://doi.org/10.21037/tlcr.2018.02.04

[5] Reck, M., Rodríguez-Abreu, D., Robinson, A.G., et al. (2019) Updated Analysis of KEYNOTE-024: Pembrolizumab versus Platinum-Based Chemotherapy for Advanced Non-Small-Cell Lung Cancer with PD-L1 Tumor Proportion Score of 50\% or Greater. Journal of Clinical Oncology, 37, 537-546. https://doi.org/10.1200/jco.18.00149

[6] (2018) Pembrolizumab plus Chemotherapy in Lung Cancer [Internet]. New England Journal of Medicine, 379, e18. http://www.nejm.org/doi/10.1056/NEJMc1808567

[7] Paz-Ares, L., Luft, A., Vicente, D., et al. (2018) Pembrolizumab plus Chemotherapy for Squamous Non-Small-Cell Lung Cancer [Internet]. New England Journal of Medicine, 379, 2040-2051. http://www.nejm.org/doi/10.1056/NEJMoa1810865 https://doi.org/10.1056/nejmoa1810865

[8] Reck, M., Schenker, M., Lee, K.H., et al. (2019) Nivolumab plus Ipilimumab versus Chemotherapy as First-Line Treatment in Advanced Non-Small-Cell Lung Cancer with High Tumour Mutational Burden: Patient-Reported Outcomes Results from the Randomised, Open-Label, Phase III CheckMate 227 Trial. European Journal of Cancer, 116, 137-147. https://doi.org/10.1016/j.ejca.2019.05.008 
[9] Lisberg, A., Cummings, A., Goldman, J.W., et al. (2018) A Phase II Study of Pembrolizumab in EGFR-Mutant, PD-L1+, Tyrosine Kinase Inhibitor Naïve Patients with Advanced NSCLC [Internet]. Journal of Thoracic Oncology, 13, 1138-1145.

http://www.ncbi.nlm.nih.gov/pubmed/29874546

https://doi.org/10.1016/j.jtho.2017.09.456 